\title{
Tip-to-tip interaction in the crystal packing of PACSIN 2 is important in regulating tubulation activity
}

\author{
Xiaoyun Bai ${ }^{1,2}$, Xiaofeng Zheng ${ }^{1,2 \bowtie}$ \\ ${ }^{1}$ State Key Lab of Protein and Plant Gene Research, School of Life Sciences, Peking University, Beijing 100871, China \\ 2 Department of Biochemistry and Molecular Biology, School of Life Sciences, Peking University, Beijing 100871, China \\ $\triangle$ Correspondence: xiaofengz@pku.edu.cn \\ Received May 11, 2013 Accepted July 5, 2013
}

\begin{abstract}
The F-BAR domain containing proteins PACSINs are cytoplasmic phosphoproteins involved in various membrane deformations, such as actin reorganization, vesicle transport and microtubule movement. Our previous study shows that all PACSINs are composed of crescent shaped dimers with two wedge loops, and the wedge loopmediated lateral interaction between neighboring dimers is important for protein packing and tubulation activity. Here, from the crystal packing of PACSIN 2, we observed a tight tip-to-tip interaction, in addition to the wedge loopmediated lateral interaction. With this tip-to-tip interaction, the whole packing of PACSIN 2 shows a spiral-like assembly with a central hole from the top view. Elimination of this tip-to-tip connection inhibited the tubulation function of PACSIN 2, indicating that tip-to-tip interaction plays an important role in membrane deformation activity. Together with our previous study, we proposed a packing model for the assembly of PACSIN 2 on membrane, where the proteins are connected by tip-to-tip and wedge loop-mediated lateral interactions on the surface of membrane to generate various diameter tubules.
\end{abstract}

KEYWORDS PACSIN 2, crystal packing, tip-to-tip interaction, tubulation, wedge loop

\section{INTRODUCTION}

The F-BAR domain containing proteins PACSINs are cytoplasmic phosphoproteins functioned in different membrane deformation activities (Damke et al., 1994; Hinshaw and Schmid, 1995; Takei et al., 1995, 1999), such as actin reor- ganization, microtubule movement (Modregger et al., 2000; Qualmann and Kelly, 2000; Braun et al., 2005; Kessels et al., 2006; Grimm-Gunter et al., 2008), as well as many other traffic events occurring at intracellular sorting compartments (Halbach et al., 2007).

The PACSINs contain neuron specific PACSIN 1, the ubiquitously expressed PACSIN 2, and the lung and muscle specific PACSIN 3 (Plomann et al., 1998; Qualmann et al., 1999; Ritter et al., 1999; Modregger et al., 2000), which all contain an $\mathrm{N}$-terminal F-BAR domain and a C-terminal SH3 domain. Recent studies show that PACSIN 2 is involved in many important pathways and diseases. For example, PACSIN 2 regulates epidermal growth factor receptor internalization (de Kreuk et al., 2012; Stocco et al., 2012b) and is correlated with lymphoblastic leukemia disease in children (Stocco et al., 2012a). PACSIN 2 is recruited to caveolae and functions in caveolar biogenesis (Hansen et al., 2011; Senju et al., 2011; Koch et al., 2012), participates in receptor-mediated endocytosis and actin organization (Qualmann and Kelly, 2000). PACSIN 2 is also shown to regulate cell spreading, migration and cell adhesion (Cousin et al., 2008; de Kreuk et al., 2011), and promote microtubule assembly (Grimm-Gunter et al., 2008). Compared with other F-BAR domain proteins such as FBP17 and CIP4, PACSIN 2 can generate tubules with less rigidity (Tanaka-Takiguchi et al., 2013). The function of PACSIN 2 in cellular events is mostly correlated with its membrane deformation ability. Packing of F-BAR proteins on membrane is very important for their membrane deformation activities (Frost et al., 2008). However, the detail mechanism of how PACSIN 2 packs on the membrane is still not clear.

Our previous study showed that all PACSINs adopt crescent shaped dimers with two unique wedge loops (Plomann et al., 2010; Shimada et al., 2010; Bai et al., 2012). Neighboring 
dimers of PACSINs are connected by wedge loop-mediated lateral interactions and form filaments on membrane to generate various tubules. The diameters of the induced tubules are correlated with the rotation angles between neighboring dimers (Bai et al., 2012). In this study, further analysis of the structure of PACSIN 2 revealed a tip-to-tip connection mode, in addition to the wedge loop-mediated lateral interaction. Both mutagenesis and tubulation assays showed that elimination of this tipto-tip interaction inhibits the tubulation function of PACSIN 2 by affecting the number, diameter, and the length of tubules. In addition, the top view of PACSIN 2 crystal packing shows a spiral-like assembly with a central hole, with the positive charged residues inside the hole, indicating that the negatively charged tubules bind the positively charged residues on the inner surface. Taken together, our studies suggest that both tipto-tip interaction and wedge loop-mediated lateral connection play important roles in regulating the tubulation activity of PACSIN 2. All these interactions confer the PACSINs' dynamically packing on membrane and further generating different degrees of membrane curvatures.

\section{RESULTS}

\section{Overall structure of PACSIN 2}

Crystals of PACSIN 2 were obtained within one week from the condition of $100 \mathrm{mmol} / \mathrm{L} \mathrm{MgCl}_{2}, 100 \mathrm{mmol} / \mathrm{L}$ sodium cacodylate, $\mathrm{pH}$ 6.5, 18\% PEG $3350(w / v)$ at $288 \mathrm{~K}$. Data collection was performed with $30 \%$ glycerol as the cryprotectant. The crystal diffracted at $2.6 \AA$ with $a=31.58, b=86.13, c=353.80$, $\alpha=90^{\circ}, \beta=90.02^{\circ}, \gamma=90^{\circ}$, which belongs to space group $P 2_{1}$ (Bai et al., 2012).

The structure of PACSIN 2 (PDB code 3Q0K) was solved by molecular replacement using the initial structure of the PACSIN 1 F-BAR domain as the searching model. Unlike the PACSIN 1 or 3 with one dimer in each unit cell, the crystal structure of PACSIN 2 is shown to consist of one tetramer with two neighboring dimers. Each dimer adopts a crescent shape with positively charged surface on the bottom with 67 lysines, 34 arginines and 16 histidines (Fig. 1B). In each dimer, two wedge loops are observed between helices $\alpha 2$ and $\alpha 3$ with the residues ranging from His119 to Glu130. One of the wedge loops mediates the neighboring dimer interaction within the tetramer through the interactions between residues in the wedge loop (His121 and Lys129) and residues of the adjacent dimer (Glu91, Glu95, and Glu99) (Fig. 1C). The other wedge loop directed toward the bottom to bind to the liposome by the hydrophobic residues, which is similar to that in PACSIN 1 and 3 (Bai et al., 2012). The monomer in each dimer is composed of a bundle core with a helix 1 (residue 24-73), a helix 2 (residue 76-120), $\alpha$ helix 5 (residue 263-275) and $\alpha$ helix 6 (residue 278-291). Two helix bundle arms of a helix 3 (residue 128-177) and $\alpha$ helix 4 (residue 187-260) extend from the central body with a curved end, respectively.
Tip-to-tip interactions in the packing of PACSIN 2

From the crystal packing of PACSIN 2, we found that in addition to the wedge loop-mediated lateral interaction (Fig. 1C), PACSIN 2 proteins are also connected by tip-to-tip interactions through ionic bonds or hydrogen bonds formed between residue Lys177 to Asp179, residue Pro184 to Leu187, residue Asn183 to Leu187, and residue Glu185 to Lys188 (Fig. 3). With this tip-to-tip interaction, the packing of PACSIN 2 shows a spiral-like assembly with a central core from the top view (Fig. 2A). The inside of the central hole is mainly consist of positively charged residues which come from the bottom surface of each dimer with 67 lysines, 34 arginines and 16 histidines (Fig. 2B), indicating that the inner surface of the hole may contribute to the binding of the negatively charged tubules.

\section{Function of tip-to-tip interactions in PACSIN 2 tubulation activity}

Because packing of BAR-domain proteins on membrane is very important for membrane remodeling, we speculated that this tip-to-tip connection also plays key functions in PACSIN assembly and membrane tubulation activity. To verify our prediction, we mutated the residues Asp179, Asn183, Pro184, Glu185, and Leu187 that are involved in tip-to-tip interactions. Mutant proteins were purified and incubated with liposome containing 20\% DOPA and 80\% DOPC as previously reported (Bai et al., 2012). Tubulation activities of these mutations were examined by transmission electron microscope (Fig. 4A) and the results were summarized in Fig. 4B. The liposome tubulation activity was completely abolished in the mutants of $\mathrm{P} 184 \mathrm{~A}$, E185K, and L187A. In the case of N183A and D179K mutants, although the liposome tubulation activity was retained, the numbers of tubules were largely decreased and the mutants could only induce $20-50 \mathrm{~nm}$ diameter tubules with the length ranging from $100 \mathrm{~nm}$ to $200 \mathrm{~nm}$, whereas the wild-type PACSIN 2 could generate tubules with various diameters $(10-200 \mathrm{~nm})$ (Bai et al., 2012) with the length ranging from $1 \mu \mathrm{m}$ to $3 \mu \mathrm{m}$ (Fig. 4). The phenotypes of mutation constructs indicate that residues Asn183, Asp179, Pro184, Glu185, and Leu187 form tight connections in the surface of tip-to-tip interaction area of PACSIN 2, which further contribute to the packing of PACSIN 2 on the membrane to induce liposome tubulation.

In addition, to investigate whether the loss of the tubulation activity is caused by the destroying of the protein structure, we examined the secondary structure of all these mutants by performing circular dichroism spectra measurement. Both the wild-type and mutants of PACSIN 2 F-BAR proteins displayed evident negative absorption at $208 \mathrm{~nm}$ and $222 \mathrm{~nm}$ with $\alpha$-helix and showed similar pattern of CD spectra (Fig. 4C), indicating that the loss of the tubulation activity is caused by the elimination of tip-to-tip interaction, but not due to the destruction of the secondary structure of PACSIN 2 protein. 
A

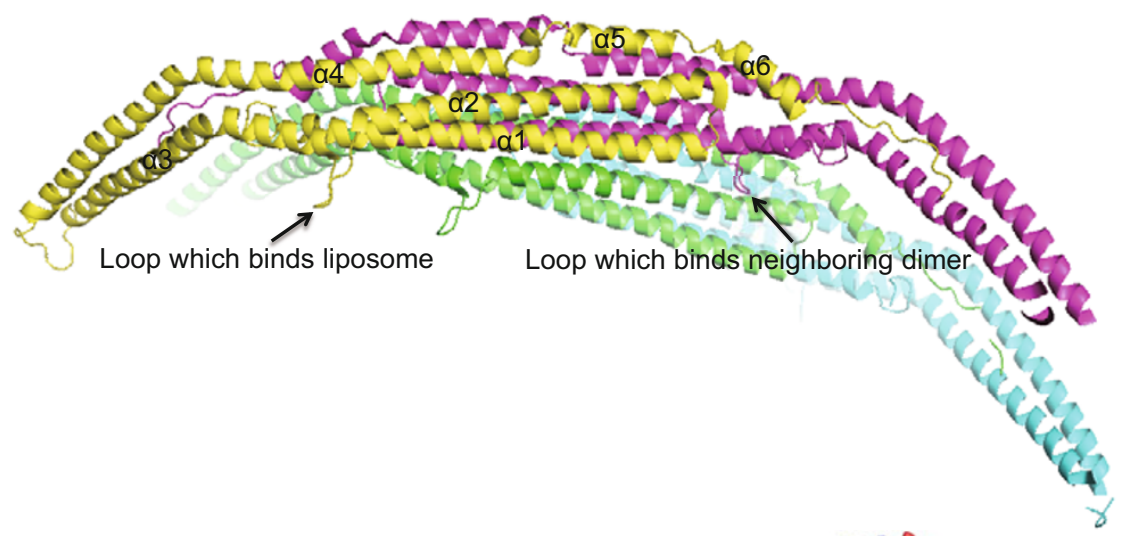

B
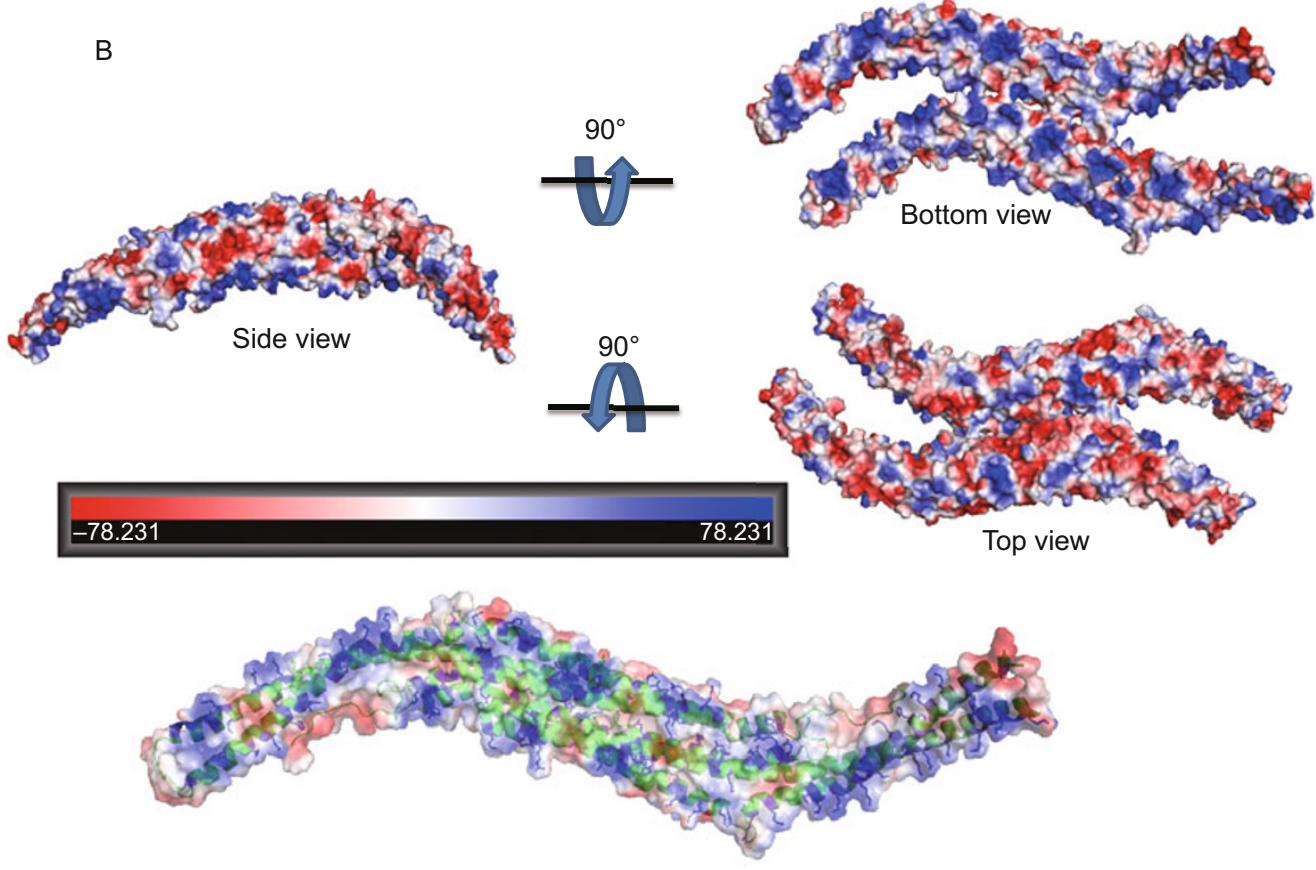

C

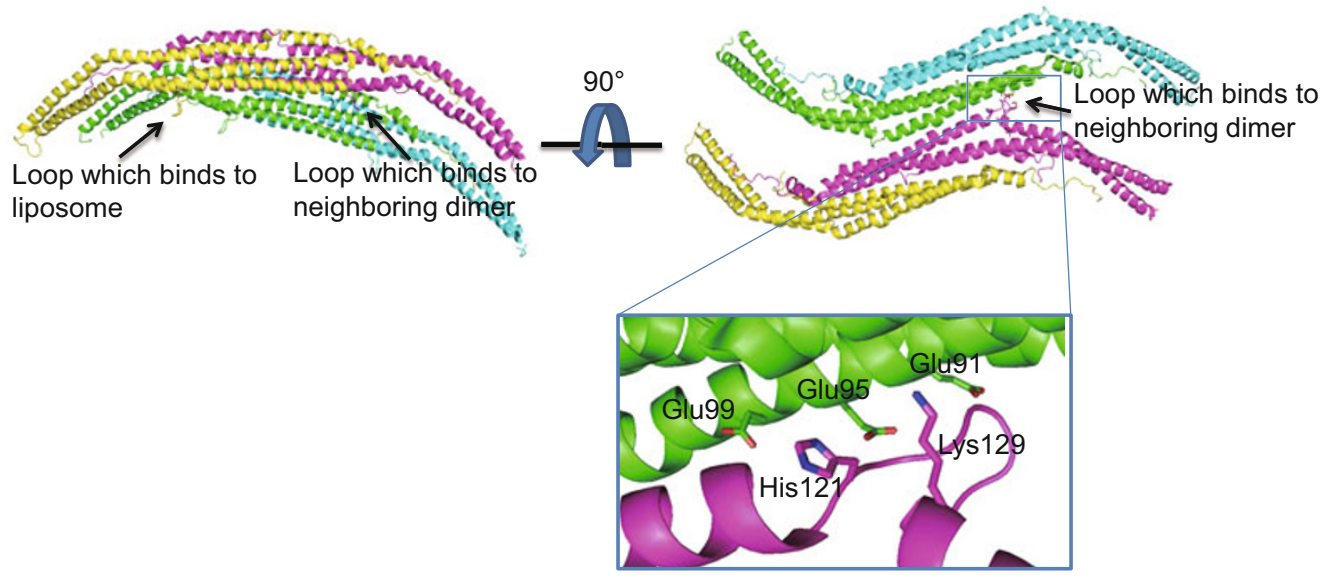

Figure 1. Overall structure of PACSIN 2 tetramer. (A) Overall structure of human PACSIN 2 tetramer. The four monomers are colored in yellow, magenta, green and cyan, respectively. Arrows indicate the two wedge loops involved in liposome binding and neighboring dimer interaction, respectively. (B) Electrostatic potential of the PACSIN 2 F-BAR domain. Basic regions are shown in blue, and the acidic regions are shown in red. Lowest panel shows that each dimer adopts a crescent shape with a positively charged surface on the bottom with 67 lysines, 34 arginines and 16 histidines. Positively charged residues are shown by side chain sticks and colored with blue. Electrostatic surface is shown with $40 \%$ transparency. (C) Arrows indicate that one wedge loop (yellow) exposing into the target membrane direction, and the other wedge loop (pink) interacts with its adjacent dimer. The contacting area is represented as side chain sticks. Inset showed the higher magnifications of the contacting area within the tetramer mediated by residues indicated. 


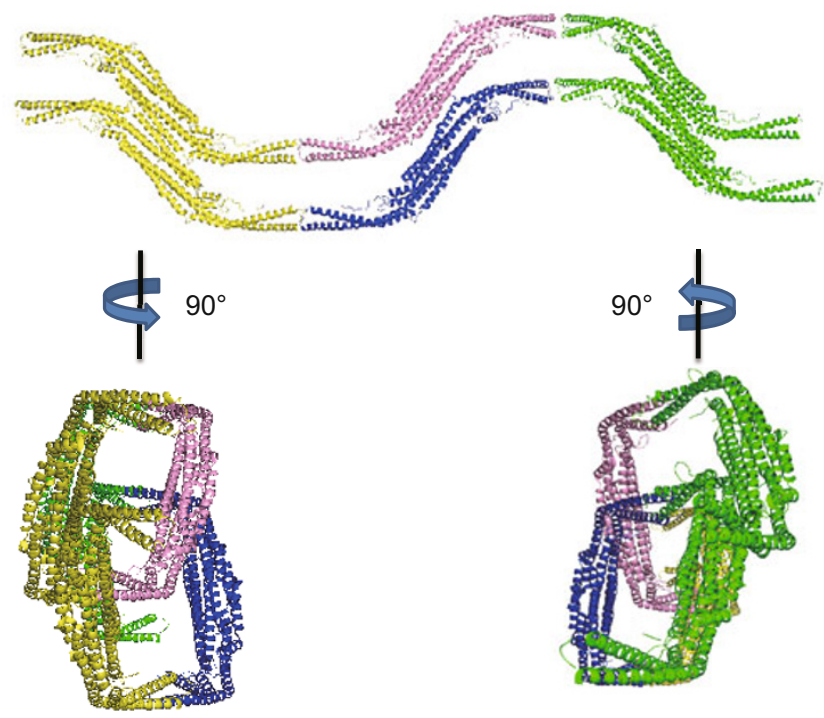

B

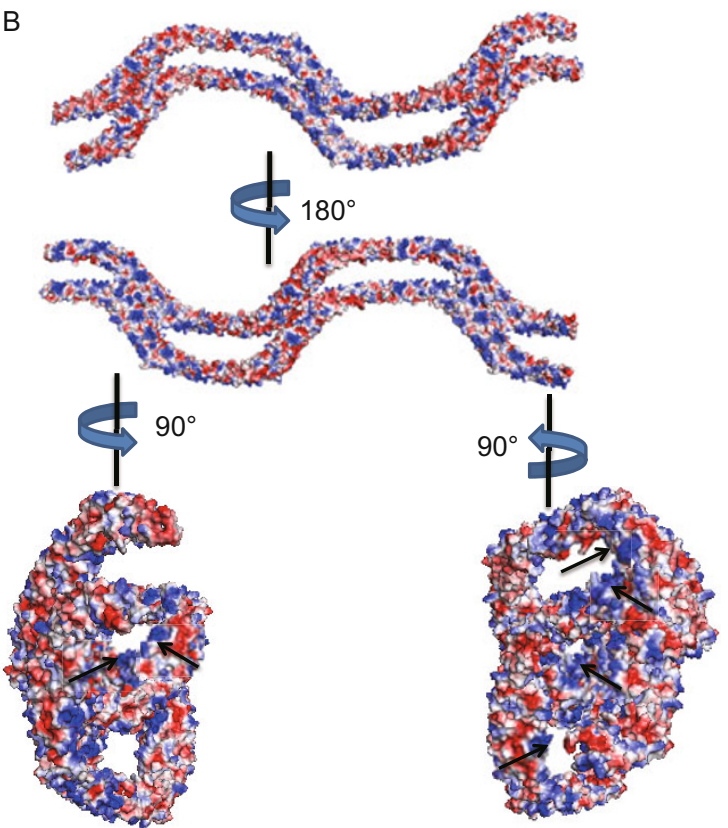

Figure 2. Spiral like assembly of PACSIN 2 packing. (A) The side view of PACSIN 2 packing (upper panel). Central neighboring dimers are colored in blue and pink. The other two connecting tetramers are colored in yellow and green, respectively. Top view of PACSIN 2 packing with a central hole (lower panel). (B) Electrostatic potential of the spiral like assembly. Basic regions of PACSIN 2 and the acidic regions are colored in blue and red, respectively.
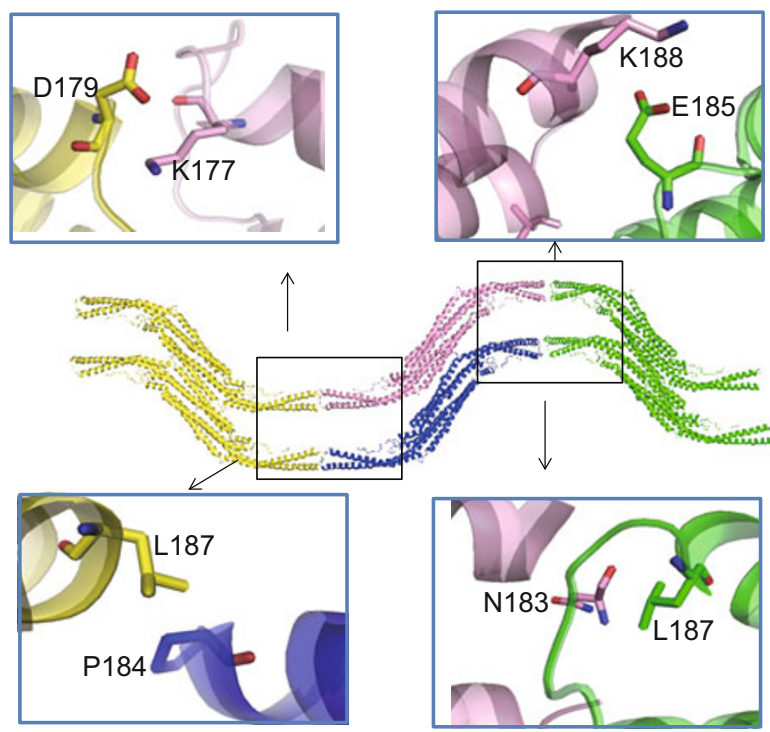

Figure 3. Close-up view of the tip-to-tip contacting area residues by Asp179-Lys177, residue Pro184-Leu187, residue Asn183-Leu187, and residue Glu185-Lys188. Interacting residues are shown as sticks.

\section{DISCUSSION}

PACSINs all contain two wedge loops, with one wedge loop involved in neighboring dimer interaction and the other loop binds liposome through its hydrophobic residues (Bai et al., 2012). PACSINs bind to liposomes through both positively charged residues on the bottom surface and hydrophobic residues in the wedge loop (Wang et al., 2009; Bai et al., 2012). Neighboring dimers of all PACSINs are connected side-by-side by the wedge loop and the diameters of the tubules are correlated with the angles between neighboring dimers (Bai et al., 2012). Analysis of the crystal packing of PACSIN 2 here further showed that the tip-to-tip interaction may also contribute to the PACSIN 2 packing on membrane. We thus mutated the residues on the surface of tip-to-tip interaction and examined their effects on tubulation activity. Compared with the F-BAR of wildtype PACSIN 2, mutants in the tip-to-tip contacting area largely decrease the number of tubules, and change the diameter and the length of the tubules as well. In addition, we found that the B-factor in the distal ends of PACSIN 2 is relatively higher compared with the average level, suggesting that the tip-to-tip connection does not restrict to certain fixed residues. The tip-to-tip contacting residues may dynamically change their interacting partners during the movement of proteins on membrane, which helps to confer the membrane deformation ability of PACSIN 2.

According to the crystal packing pattern of PACSIN 2 and the mutagenesis studies, we proposed a model of how PACSIN 2 functions on the membrane: PACSIN 2 proteins are connected by wedge loop-mediated lateral interaction and tip-to-tip interaction (Fig. 5), and bind to liposome through the positively charged surface and the hydrophobic residues in the wedge loop. All the dimers tune around the membrane to form the cor- 
A

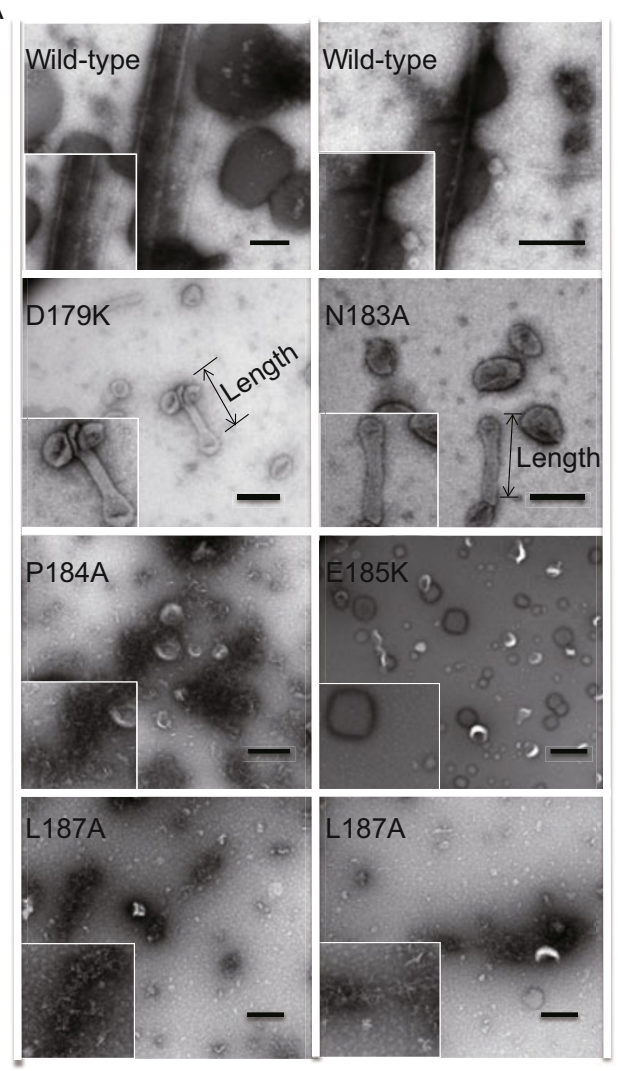

\begin{tabular}{|c|c|c|c|}
\hline Name & Tubule number & Diameter & Tubule length \\
\hline Wild-type & ++++ & $10-200 \mathrm{~nm}$ & $1-3 \mu \mathrm{m}$ \\
\hline D179K & + & $20-50 \mathrm{~nm}$ & $100-200 \mathrm{~nm}$ \\
\hline N183A & + & $20-50 \mathrm{~nm}$ & $100-200 \mathrm{~nm}$ \\
\hline P184A & 0 & - & - \\
\hline E185K & 0 & - & - \\
\hline L187A & 0 & - & - \\
\hline
\end{tabular}

C

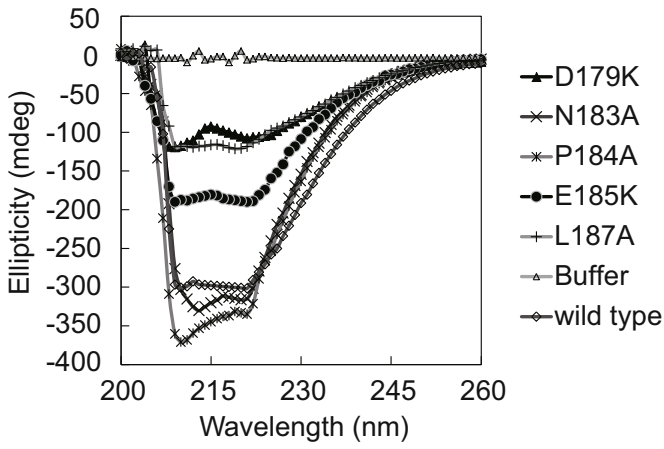

Figure 4. Tubulation activities of wild-type and mutants of PACSIN 2 F-BAR domain. Insets show the higher magnifications of the tubules indicated by arrows. Scale bars, $100 \mathrm{~nm}$. (B) Summary of the tubulation phenotype. "+" indicates that the average number of tubules is less than 5, whereas "++++" indicates that the average number of tubules is more than 20. (C) The secondary structures of the PACSIN 2 mutants were detected by Circular Dichroism analysis. The characteristic shape and magnitude of the CD spectral trace are all indicative of $\alpha$-helix conformation. Three scans were averaged for wild-type protein or each mutant. "Wild type protein" indicates PACSIN 2 F-BAR protein. "Buffer" indicates the buffer containing $10 \mathrm{mmol} / \mathrm{L} \mathrm{HEPES} \mathrm{pH} 7.5,100 \mathrm{mmol} / \mathrm{L} \mathrm{NaCl}$.

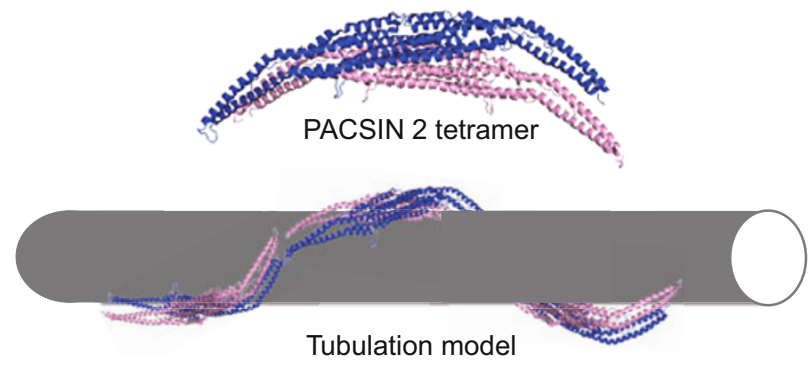

Figure 5. Model of PACSIN 2 packing on membrane. The neighboring dimers are colored in blue and pink and shown as ribbon, respectively. The three tetramers are connected by tip-totip interactions and generated the tubules by binding the liposome with their positively charged surface, as well as the hydrophobic residues in the wedge loop.

responding tubules with various diameters. This model helps us to further understand the packing mode of PACSIN family on membrane.
In F-BAR domain containing proteins, both lateral and tipto-tip contacting modes have been observed in CIP4 and FBP17 (Frost et al., 2008). Here the study on PACSIN 2 further indicates that these two kinds of interactions are key factors that affect the tubulation activities of F-BAR proteins. However, the distinct characteristics of PACSINs that are different from other F-BAR proteins are that the lateral connections are mediated by the unique wedge loop, and the diameter of induced tubules is not correlated with the intrinsic curvature of the F-BAR domain, but associated with the whole packing of proteins on membrane. Further direct view of how PACSINs pack on membrane by Cryo-EM is necessary in the future.

\section{MATERIALS AND METHODS}

Cloning and protein expression

F-BAR domain of PACSIN 2 (residue 1-341) was cloned into the $B a m \mathrm{HI}$ and Hindll sites of pET28a vector and confirmed by DNA sequencing. Mutants of D179K, N183A, P184A, E185K, and L187A were constructed into $\mathrm{pET} 28 \mathrm{a}$ vector by site-directed mutagenesis methods 
Table 1. Crystallographic parameters and data-collection statistics of PACSIN 2

\begin{tabular}{ll}
\hline Data collection & \\
\hline Wavelength $(\AA)$ & 1.072 \\
Resolution $(\AA)$ & $30-2.6 \AA(2.75-2.6 \AA)$ \\
Completeness $(\%)$ & $99.7(85.9)$ \\
Mean I/Sig I & $14.5(2.5)$ \\
Space group & $\mathrm{P} 2{ }_{1}$ \\
Unit-cell parameters $(\AA)$ & $\mathrm{a}=31.58, \mathrm{~b}=86.13, \mathrm{C}=353.80$ \\
& $\alpha=90^{\circ}, \beta=90.02^{\circ}, \mathrm{Y}=90^{\circ}$ \\
\hline Refinement & \\
\hline Protein atoms & 9523 \\
Solvent atoms & 202 \\
$\mathrm{R}_{\text {merge }} / \mathrm{R}_{\text {free }}(\%)$ & $20.77(29.2)$ \\
Bonds $(\AA)$ & 0.015 \\
Angles $\left(^{\circ}\right)$ & 1.593 \\
Unique reflections & 41646 \\
Multiplicity & 3.1 \\
Mol/Asym & 4 \\
\hline
\end{tabular}

Figures in brackets: outer shell data.

$R_{\text {free }}=\Sigma|| F_{\mathrm{o}}|-| F_{\mathrm{c}}|| / \Sigma\left|F_{\mathrm{o}}\right|$

using the cDNA of PACSIN 2 as template, and verified by DNA sequencing. The proteins were expressed in Escherichia coli strain BL21 (DE3) and purified on a $\mathrm{Ni}^{2+}$-HiTrap affinity column and a Superdex-75 column (GE Healthcare) as previously reported (Bai et al., 2012).

\section{Diffraction data collection}

Protein was crystallized and optimized by sitting drop and hanging drop, respectively (Bai et al., 2012). For crystal data collection, crystals were soaked in the corresponding cryoprotectant solution which consists of the reservoir solution $80 \%(\mathrm{~V} / \mathrm{V})$ and $20 \%(\mathrm{~V} / \mathrm{V})$ ethylene glycol. The crystal was then mounted in a cryoloop and flash-cooled in a nitrogen stream at $100 \mathrm{~K}$. X-ray diffraction data were collected on a MAR 345 image-plate detector at Beijing Synchrotron Radiation Facility (beamline 3W1A), Institute of High Energy Physics, Chinese Academy of Life Sciences. A total of 720 frames of $0.5^{\circ}$ oscillation were measured with $60 \mathrm{~s}$ exposure per frame (Table 1). Data were processed with HKL2000 (Z Otwinowski, 1997) and CCP4 (Collaborative Computational Project, 1994).

\section{Liposome preparation}

As reported in our previous study (Bai et al., 2012), lipids containing 80\% DOPC and 20\% DOPA (Avanti) were treated in chloroform and nitrogen gas, and incubated for $2 \mathrm{~h}$ in a vacuum to completely remove the solvent. Lipid films were resuspended and subjected to 10 freezethaw cycles, then filtered by extrusion through $100 \mathrm{~nm}$ nucleopore polycarbonate membranes.

\section{Tubulation assays}

Tubulation analyses were performed following the procedure described in our previous study (Bai et al., 2012). Briefly, the purified protein
$(1 \mathrm{mg} / \mathrm{mL})$ was incubated with liposome $(1 \mathrm{mg} / \mathrm{mL})$ with $1: 1$ proteinlipid volume ratio for $5 \mathrm{~min}$ at room temperature. The mixtures were then spread onto freshly glow-discharged formvar- and carbon-coated electron microscopy grids, stained with $2 \%$ uranyl acetate. The grid was finally examined on a transmission electron microscope (FEI 200 $\mathrm{kV}$ ) with the electron energy set to $120 \mathrm{kV}$.

\section{ACCESSION CODE}

Protein Data Bank: Atomic coordinates and structure factors for PACSIN 2 F-BAR domain have been deposited with the accession codes 3QOK.

\section{ACKNOWLEDGEMENTS}

We are grateful to Prof. Fuyu Yang and Dr. Hejiang Zhou from Institute of Biophysics, Chinese Academy of Sciences, for lipid preparation. We thank Prof. Yicheng Dong for helpful discussion. X-ray diffraction data collection was carried out at the Beijing Synchrotron Radiation Laboratory and Beijing Synchrotron Radiation Facility. This work was supported by grants from the National High Technology and Development Program of China (973 Programs) (No. 2010CB911800) and the National Natural Science Foundation of China (Grant No.30930020).

\section{ABBREVIATIONS}

BAR proteins, Bin/amphiphysin/Rvs domain proteins; PACSIN, Protein kinase $C$ and casein kinase substrate in neurons protein

\section{COMPLIANCE WITH ETHICS GUIDELINES}

Xiaoyun Bai and Xiaofeng Zheng declare that they have no conflict of interest.

This article does not contain any studies with human or animal subjects performed by the any of the authors.

\section{REFERENCES}

Bai, X., Meng, G., Luo, M., and Zheng, X. (2012). Rigidity of wedge loop in PACSIN 3 protein is a key factor in dictating diameters of tubules. J Biol Chem 287, 22387-22396.

Braun, A., Pinyol, R., Dahlhaus, R., Koch, D., Fonarev, P., Grant, B.D., Kessels, M.M., and Qualmann, B. (2005). EHD proteins associate with syndapin I and II and such interactions play a crucial role in endosomal recycling. Mol Biol Cell 16, 3642-3658.

Collaborative Computational Project, N. (1994). The CCP4 suite: programs for protein crystallography. Acta Crystallogr D Biol Crystallogr 50, 760-763.

Cousin, H., Desimone, D.W., and Alfandari, D. (2008). PACSIN2 regulates cell adhesion during gastrulation in Xenopus laevis. Dev Biol 319, 86-99.

Damke, H., Baba, T., Warnock, D.E., and Schmid, S.L. (1994). Induction of mutant dynamin specifically blocks endocytic coated vesicle formation. J Cell Biol 127, 915-934.

de Kreuk, B.J., Anthony, E.C., Geerts, D., and Hordijk, P.L. (2012). The F-BAR protein PACSIN2 regulates epidermal growth factor receptor internalization. J Biol Chem 287, 43438-43453.

de Kreuk, B.J., Nethe, M., Fernandez-Borja, M., Anthony, E.C., Hensbergen, P.J., Deelder, A.M., Plomann, M., and Hordijk, P.L. (2011). 
The F-BAR domain protein PACSIN2 associates with Rac1 and regulates cell spreading and migration. J Cell Sci 124, 2375-2388.

Frost, A., Perera, R., Roux, A., Spasov, K., Destaing, O., Egelman, E.H., De Camilli, P., and Unger, V.M. (2008). Structural basis of membrane invagination by F-BAR domains. Cell 132, 807-817.

Grimm-Gunter, E.M., Milbrandt, M., Merkl, B., Paulsson, M., and Plomann, M. (2008). PACSIN proteins bind tubulin and promote microtubule assembly. Exp Cell Res 314, 1991-2003.

Halbach, A., Morgelin, M., Baumgarten, M., Milbrandt, M., Paulsson, M., and Plomann, M. (2007). PACSIN 1 forms tetramers via its $\mathrm{N}$ terminal F-BAR domain. FEBS J 274, 773-782.

Hansen, C.G., Howard, G., and Nichols, B.J. (2011). Pacsin 2 is recruited to caveolae and functions in caveolar biogenesis. J Cell Sci 124, 2777-2785.

Hinshaw, J.E., and Schmid, S.L. (1995). Dynamin self-assembles into rings suggesting a mechanism for coated vesicle budding. Nature 374, 190-192.

Kessels, M.M., Dong, J., Leibig, W., Westermann, P., and Qualmann, B. (2006). Complexes of syndapin II with dynamin II promote vesicle formation at the trans-Golgi network. J Cell Sci 119, 1504-1516.

Koch, D., Westermann, M., Kessels, M.M., and Qualmann, B. (2012). Ultrastructural freeze-fracture immunolabeling identifies plasma membrane-localized syndapin II as a crucial factor in shaping caveolae. Histochem Cell Biol 138, 215-230.

Modregger, J., Ritter, B., Witter, B., Paulsson, M., and Plomann, M. (2000). All three PACSIN isoforms bind to endocytic proteins and inhibit endocytosis. J Cell Sci 113 Pt 24, 4511-4521.

Plomann, M., Lange, R., Vopper, G., Cremer, H., Heinlein, U.A., Scheff, S., Baldwin, S.A., Leitges, M., Cramer, M., Paulsson, M., et al. (1998). PACSIN, a brain protein that is upregulated upon differentiation into neuronal cells. Eur J Biochem 256, 201-211.

Plomann, M., Wittmann, J.G., and Rudolph, M.G. (2010). A hinge in the distal end of the PACSIN 2 F-BAR domain may contribute to membrane-curvature sensing. J Mol Biol 400, 129-136.

Qualmann, B., and Kelly, R.B. (2000). Syndapin isoforms participate in receptor-mediated endocytosis and actin organization. J Cell Biol 148, 1047-1062.

Qualmann, B., Roos, J., DiGregorio, P.J., and Kelly, R.B. (1999). Syndapin I, a synaptic dynamin-binding protein that associates with the neural Wiskott-Aldrich syndrome protein. Mol Biol Cell 10,
501-513.

Ritter, B., Modregger, J., Paulsson, M., and Plomann, M. (1999). PACSIN 2, a novel member of the PACSIN family of cytoplasmic adapter proteins. FEBS Lett 454, 356-362.

Senju, Y., Itoh, Y., Takano, K., Hamada, S., and Suetsugu, S. (2011). Essential role of PACSIN2/syndapin-II in caveolae membrane sculpting. J Cell Sci 124, 2032-2040.

Shimada, A., Takano, K., Shirouzu, M., Hanawa-Suetsugu, K., Terada, T., Toyooka, K., Umehara, T., Yamamoto, M., Yokoyama, S., and Suetsugu, S. (2010). Mapping of the basic amino-acid residues responsible for tubulation and cellular protrusion by the EFC/F-BAR domain of pacsin2/Syndapin II. FEBS Lett 584, 1111-1118.

Stocco, G., Franca, R., Verzegnassi, F., Londero, M., Rabusin, M., and Decorti, G. (2012a). Multilocus genotypes of relevance for drug metabolizing enzymes and therapy with thiopurines in patients with acute lymphoblastic leukemia. Front Genet 3, 309.

Stocco, G., Yang, W., Crews, K.R., Thierfelder, W.E., Decorti, G., Londero, M., Franca, R., Rabusin, M., Valsecchi, M.G., Pei, D., et al. (2012b). PACSIN2 polymorphism influences TPMT activity and mercaptopurine-related gastrointestinal toxicity. Human Mol Genet 21, 4793-4804.

Takei, K., Slepnev, V.I., Haucke, V., and De Camilli, P. (1999). Functional partnership between amphiphysin and dynamin in clathrinmediated endocytosis. Nat Cell Biol 1, 33-39.

Takei, Y., Harada, A., Takeda, S., Kobayashi, K., Terada, S., Noda, T., Takahashi, T., and Hirokawa, N. (1995). Synapsin I deficiency results in the structural change in the presynaptic terminals in the murine nervous system. J Cell Biol 131, 1789-1800.

Tanaka-Takiguchi, Y., Itoh, T., Tsujita, K., Yamada, S., Yanagisawa, M., Fujiwara, K., Yamamoto, A., Ichikawa, M., and Takiguchi, K. (2013). Physicochemical analysis from real-time imaging of liposome tubulation reveals the characteristics of individual F-BAR domain proteins. Langmuir 29, 328-336.

Wang, Q., Navarro, M.V., Peng, G., Molinelli, E., Goh, S.L., Judson, B.L., Rajashankar, K.R., and Sondermann, H. (2009). Molecular mechanism of membrane constriction and tubulation mediated by the F-BAR protein Pacsin/Syndapin. Proc Natl Acad Sci U S A 106, 12700-12705.

Z Otwinowski, W.M. (1997). Processing of X-ray diffraction data collected in oscillation mode. Methods Enzymol 276, 307-326. 